\title{
SATURACIÓN DE LA OXIHEMOGLOBINA Y RELACIÓN VENTRÍCULO DERECHO/VENTRÍCULO TOTAL(VD/VT) ENDOS LÍNEAS COMERCIALES DE POLLOS DE CARNE CRIADOS A NIVEL DEL MAR
}

\author{
Saturation of Oxihemoglobin and Rigth Ventricle / Total Ventricle \\ (RV/TV) Relationship in Two Commercial Lines of BroILERS \\ RAISED AT SEa LeVEL
}

Christian Salazar L. ${ }^{1}$, Sergio Cueva M. ${ }^{1}$, Boris Lira M. ${ }^{1}$, María Vásquez C. ${ }^{1}$, José Rodríguez G. . $^{1,2}$

\section{Resumen}

El estudio tuvo por objetivo determinar la relación entre el peso, saturación de la oxihemoglobina y la relación ventrículo derecho / ventrículo total (VD/VT) entre dos líneas comerciales de carnes de pollo a nivel del mar. Se utilizaron 75 pollos machos de la línea Ross (R) y 45 de la línea Cobb Vantress (CV). Las aves se pesaron en el día 1, 10, 20 30 y 40 de edad. Se determinó la saturación de la oxihemoglobina, y luego del sacrificio, se calculó la relación ventrículo derecho/ventrículo total (VD/VT) en 25 pollos R y $15 \mathrm{CV}$ los días 20, 30 y 40. Adicionalmente, se calculó el índice de conversión alimenticia. La relación VD/VT para CV fue de $0.195 \pm 0.12,0.199 \pm 0.013$ y $0.188 \pm 0.032$ y en $\mathrm{R}$ de 0.205 $\pm 0.016,0.179 \pm 0.022$ y $0.245 \pm 0.042$ a los 20,30 y 40 días, respectivamente, con diferencias estadísticas entre líneas ( $\mathrm{p}<0.05$ ). La saturación de oxihemoglobina varió entre 92.06 y $95.75 \%$ sin diferencias estadísticas entre líneas. Los pollos CV fueron más pesados que $\mathrm{R}(\mathrm{p}<0.05)$ hasta el día 30. Se concluye que en la etapa de mayor crecimiento, la línea Cobb Vantress tuvo mayor peso y una mayor relación VD/VT, mientras que la saturación de oxihemoglobina fue similar a la línea Ross, encontrándose estos valores dentro de los parámetros esperados para pollos de engorde.

Palabras clave: pollos de engorde, relación VD/VT, saturación Oxi-Hb, peso corporal

\section{Abstract}

The study aimed to determine the relationship between body weight, oxyhemoglobin saturation and right ventricle/total ventricle ratio(VD/VT) in two commercial broiler lines raised at sea level. It was used 75 chicken males of Ross line (R) and 50 of Cobb Vantress line (CV). The birds were weighed on days 1, 10, 20, 30, and 40 of age. The oxyhemoglobin

\footnotetext{
${ }^{1}$ Laboratorio de Fisiología Animal, Facultad de Medicina Veterinaria, Universidad Nacional Mayor de San Marcos, Lima

${ }^{2}$ E-mail: joserodriguezmv@gmail.com
} 
saturation was measured, and then, after slaughtering, the ventricle right/total ventricle ratio(VD/VT) in $25 \mathrm{R}$ and $15 \mathrm{CV}$ chicks on days 20, 30 and 40 was calculated. Additionally, the conversion feed index was calculated. The VD/VT ratio for CV was $0.195 \pm 0.12,0.199$ \pm 0.013 and $0.188 \pm 0.032$, and in $R$ was $0.205 \pm 0.016,0.179 \pm 0.022$ and $0.245 \pm 0.042$ on days 20, 30 and 40 respectively, with statistical difference between lines $(\mathrm{p}<0.05)$. The oxyhemoglobin saturation varied between 92.06 and $95.75 \%$ without statistical difference between lines. $\mathrm{CV}$ chicks were heavier than $\mathrm{R}(\mathrm{p}<0.05)$ until day 30 . It is concluded that in the greatest period of growth, the Cobb Vantress line showed higher body weights and major VD/VT ratio, while oxygen saturation was similar to that of chicken in the Ross line, being these values within the expected values for broilers.

Key words: broilers, VD/VT relation, Oxi-Hb saturation, body weight

\section{INTRODUCCIÓN}

Uno de los principales problemas metabólicos en aves de engorde es el Síndrome Ascítico (SA), llamado también Síndrome de Hipertensión Pulmonar (SHP), el cual es una condición patológica específica multifactorial que se caracteriza por el acúmulo de líquido en la cavidad abdominal. Su manifestación está asociada a la sobrecarga fisiológica y metabólica (Silveira et al., 2001). El SA a nivel del mar es causado por una hipoxia funcional debido al elevado requerimiento de oxígeno y la incapacidad cardiopulmonar para satisfacer la demanda de oxígeno tisular (Julián, 1993; Scheele, 1996; Wideman, 2000).

Estudios llevados a cabo en la Facultad de Medicina Veterinaria (FMV) de la Universidad Nacional Mayor de San Marcos (UNMSM) indican que la línea Ross sería posiblemente más resistente que la Cobb Vantress en desarrollar el síndrome de hipertensión arterial pulmonar (SHP) a nivel del mar, basado en que hay un menor crecimiento de la musculatura lisa de las paredes de las arteriolas pulmonares hacia el día 30 de edad (Rodríguez et al., 2012). A fin de contribuir a dilucidar lo expuesto, el presente estudio tuvo como objetivo determinar posibles diferencias entre pollos de las líneas Cobb Vantress y Ross, criados a nivel del mar, en peso corporal, niveles de saturación de la oxihemoglobina y la relación ventrículo derecho/ventrículo total (VD/VT).

\section{MATERIALes y MéTOdos}

El trabajo se realizó en las instalaciones experimentales del Laboratorio de Fisiología Animal de la FMV-UNMSM, localizada en Lima, a nivel del mar (presión barométrica de $750 \mathrm{mmHg}$ y presión parcial de oxígeno de $157 \mathrm{mmHg}$ ).

Se utilizaron 125 pollos de carne machos de líneas comerciales, siendo 50 de la línea Cobb Vantres y 75 de la línea Ross. Las aves se pesaron en los días 1, 10, 20, 30 y 40 de edad, empleando una balanza con capacidad de $5 \mathrm{~kg}$ y precisión de $1 \mathrm{~g}$. Además, se determinó la cantidad de alimento que consumieron en cada periodo de edad. Las aves fueron criadas siguiendo los estándares de crianza para producción de pollos comerciales de carne.

La saturación de hemoglobina se determinó los días 20,30 y 40 en 15 y 25 pollos, seleccionados al azar, de las líneas Cobb Vantress y Ross, respectivamente. La medición se realizó en el tercer dedo de la pata y a nivel cubital con un pulsoxímetro (Wideman et al., 2000). La zona elegida se limpió con alcohol medicinal para remover las impurezas y restos de excretas y cama. 
Cuadro 1. Peso corporal (promedio \pm d.e.) de pollos de las líneas Cobb Vantress y Ross criados a nivel del mar, según la edad

\begin{tabular}{ccc}
\hline Días de edad & Cob Vantress & Ross \\
\hline 1 & $51 \pm 4^{\mathrm{a}}$ & $47 \pm 3^{\mathrm{b}}$ \\
10 & $268 \pm 22^{\mathrm{a}}$ & $375 \pm 35^{\mathrm{b}}$ \\
20 & $811 \pm 85^{\mathrm{a}}$ & $772 \pm 66^{\mathrm{b}}$ \\
30 & $1960 \pm 240^{\mathrm{a}}$ & $1486 \pm 91^{\mathrm{b}}$ \\
40 & $2571 \pm 208^{\mathrm{a}}$ & $2727 \pm 210^{\mathrm{a}}$ \\
\hline
\end{tabular}

${ }^{a, b}$ Superíndices diferentes dentro de filas indican diferencia estadística $(\mathrm{p}<0.05)$

Las aves se sacrificaron mediante desarticulación occípito-atloidea luego de la medición de la saturación de hemoglobina. Se expuso la cavidad abdominal y se extrajo el corazón. La evaluación de este órgano fue a través de la técnica de Alexander (Alexander y Jensen, 1959). El órgano fue liberado de sus aurículas, grandes vasos, grasa circundante, elementos valvulares y coágulos. La masa ventricular fue dividida en ventrículo derecho y ventrículo izquierdo más septo interventricular. Se pesó cada sección para calcular la relación VD/VT.

Las variables VD/VT, saturación de oxihemoglobina y peso corporal fueron evaluadas mediante la prueba de T de Student para diferencia de medias.

\section{Resultados}

Los pesos corporales en los primeros 30 días de edad fueron superiores en los pollos Cobb Vantress ( $p<0.05$, Cuadro 1). Asimismo, la relación VD/VT fue estadísticamente diferente $(\mathrm{p}<0.05)$ entre líneas en todas las edades (Cuadro 2). No se halló diferencia estadística entre líneas de aves en la saturación de oxihemoglobina (Cuadro 3).

\section{Discusión}

Las aves de las dos líneas tuvieron pesos corporales similares al final del estudio (40 días de edad), aunque se observó un crecimiento inicial más acelerado en pollos de la línea Cobb Vantress ( $\mathrm{p}<0.05$, Cuadro 1), similar a otros estudios (González et al., 1998; Rodríguez et al., 2012). No obstante, trabajos previos demuestran que pollos de la línea Ross tienen un crecimiento más acelerado en etapas más tardías del crecimiento de modo que pueden igualar los pesos de la línea Cobb Vantress (Marcato et al., 2008). Si bien la línea Cobb Vantress deposita proteína en forma de músculo antes que la línea Ross, después del punto de inflexión (punto máximo en la curva de crecimiento), su tasa de deposición de músculo se vuelve más lenta, a diferencia de la línea Ross que deposita músculo más rápidamente. Según Kessler et al. (2000), cuanto mayor y más larga es la meseta de la deposición de proteínas, más eficiente es el ave en la producción de carne, y mejor será la composición de la canal.

La relación VD/VT en ambas líneas genéticas se encuentra dentro de los rangos de normalidad (Wideman et al., 2000). Julián et al. (1992) señala que valores VD/VT in- 
Cuadro 2. Relación ventrículo derecho / ventrículo total (VD/VT) de pollos de las líneas Cobb Vantress y Ross criados a nivel del mar (promedio \pm d.e.), según la edad

\begin{tabular}{ccc}
\hline Días de edad & Cob Vantress & Ross \\
\hline 20 & $0.195 \pm 0.120^{\mathrm{a}}$ & $0.205 \pm 0.016^{\mathrm{b}}$ \\
30 & $0.199 \pm 0.013^{\mathrm{a}}$ & $0.179 \pm 0.022^{\mathrm{b}}$ \\
40 & $0.188 \pm 0.032^{\mathrm{a}}$ & $0.245 \pm 0.042^{\mathrm{b}}$ \\
\hline
\end{tabular}

${ }^{a, b}$ Superíndices diferentes dentro de filas indican diferencia estadística $(p<0.05)$

Cuadro 3. Saturación de oxihemoglobina en pollos de las líneas Cobb Vantress y Ross criados a nivel del mar (promedio \pm d.e.), según la edad

\begin{tabular}{ccc}
\hline Días de edad & Cob Vantress. & Ross \\
\hline 20 & $95.07 \pm 4.23^{\mathrm{a}}$ & $94.52 \pm 4.61^{\mathrm{a}}$ \\
30 & $92.06 \pm 2.42^{\mathrm{a}}$ & $94.29 \pm 4.59^{\mathrm{a}}$ \\
40 & $95.75 \pm 3.49^{\mathrm{a}}$ & $95.57 \pm 3.11^{\mathrm{a}}$ \\
\hline
\end{tabular}

${ }^{a, b}$ Superíndices diferentes dentro de filas indican diferencia estadística $(p<0.05)$

feriores a 0.25 son normales, debiéndose sospechar de una moderada hipertrofia cuando están entre 0.25 y 0.29 y de una hipertrofia severa cuando son superiores a dicho valor. No obstante, la línea Cobb Vantress presenta una relación VD/VT mayor que la línea Ross, lo cual guarda concordancia con el aumento de grosor de la musculatura de las arteriolas pulmonares hallado por otros autores (Staub, 1963; Rodríguez et al., 2012).

En los días 20 y 30 del estudio, las aves de la línea Cobb Vantress ganaron más peso y tuvieron una mayor relación VD/VT que las aves de la línea Ross. Según Mack y Donald (1998), la curva de crecimiento del pollo de engorde es ascendente hasta la semana 7 (49 días), siendo acelerada entre la tercera y quinta semana (21-35 días). Por su parte, Hernández (1986) menciona que entre la tercera y quinta semana de edad (21-35 días) es donde hay mayor presentación de hipertensión arterial pulmonar en broilers. En el día 40 presente estudio, las tendencias iniciales variaron toda vez que la línea Ross presentó una mayor relación VD/VT y obtuvieron una mayor aunque no significativa ganancia de peso que la línea Cobb Vantress.

En base a estos resultados, se podría deducir entonces que hay una relación directa entre el aumento del metabolismo (hipoxia metabólica), producto de una mayor fijación de proteínas corporales en forma de músculo y el incremento del valor de la relación VD/VT. Esto es señalado por Marcato et al. (2008) quienes hallaron una máxima fijación de proteínas corporales alrededor del día 30 de edad en aves de la línea Cobb Vantress. Estos datos indicarían que la línea Cobb Vantress podría ser más susceptible que la línea Ross a presentar algún desbalance metabólico en la etapa de crecimiento acelerado. 
Los valores de saturación de oxihemoglobina se encuentran de los rangos normales, y similares a los reportados por Wideman et al. (2000) en pollos de engorde.

\section{Conclusiones}

- En la etapa de mayor crecimiento, las aves de la línea Cobb Vantress lograron mayores pesos corporales y una mayor relación ventrículo derecho/ventrículo total (VD/VT); mientras que la saturación de oxihemoglobina fue similar al de las aves de la línea Ross.

- Los valores obtenidos en las variables en estudio estuvieron dentro de los parámetros normales para pollos de engorde.

\section{Literatura Citada}

1. Alexander A, Jensen F. 1959. Gross cardiac changes in cattle with high mountain (brisket) disease in experimental cattle maintained at high altitudes. Am J Vet Res 20: 680-689.

2. González E, Buyse J, Sayuri T, Sartori J, Decuypere E. 1998. Metabolic disturbances in male broilers of different strains. Performance, mortality, and right ventricular hypertrophy. Poultry Sci 77: 1646-1653.

3. Hernández V. 1986. La ascitis hipóxica en pollos: Influencia y posibles soluciones. Memorias VI Seminario Internacional de Patología Aviar. Gorgia, EEUU. $\mathrm{p} 95$.

4. Julián R, Caston L, Leeson S. 1992. The effect of dietary sodium on right ventricular failure induced ascites, gain and fat deposition in meat type chickens. Can J Vet Res 56: 214-219.

5. Julian R. 1993. Ascites in poultry. Avian Pathol 22: 419-454.
6. Kessler A, Snizek PN, Brugalli I. 2000. Manipulaçao da quantidade de gordura na carcaça de frangos. Anais da conferencia Apinco de Ciência e Tecnologia Avícolas. Sâo Paulo. Brasil. Campinas: FACTA. p 107-133.

7. Mack O, Donald D. 1998. Manual de producción avícola. $4^{\mathrm{a}}$ ed. Mexico DF: Ed Manual Moderno. 829 p.

8. Marcato S, Sakomura N, Munari D, Fernandes J, Kawauchi I, Bonato M. 2008. Growth and body nutrient deposition of two broiler commercial genetic lines. Braz J Poultry Sci 10: 117123.

9. Rodríguez J, Vásquez M, Cueva S, Ayón M, Lira B, Chavera A, Angulo P, Falcón N. 2012. Comparación histológica del espesor de la capa media de las arteriolas pulmonares en pollos de carne Cobb Vantress y Ross expuestos a hipoxia. Rev Inv Vet Perú 23(2): 119125.

10. Scheele C. 1996. Ascites in chickens. Oxygen consumption and requirement related to its occurrence. In: Health and adaptation of broilers to a changed environment. The Netherlands: Netherlands Publishers. p 120-124.

11. Silveira V, Mazzuco H, Rosa P, Fiorentin L. 2001. Sindrome da hipertensao pulmonar a ascite em frangos de corte. Circular Técnica. Brasil: Ministerio de Agricultura. $27 \mathrm{p}$.

12. Staub N. 1963. Site of action of hypoxia on the pulmonary vasculature. Federation Proceedings 22: 450-453.

13. Wideman R. 2000. Pathophysiology of heart/lung disorders. XXI International Poultry Congress. Montreal, Canada. $\mathrm{p}$ 11-20.

14. Wideman R, Fedde M, Tackett C, Weiglet G. 2000. Cardio-pulmonary function in preascitic (hypoxemic) or normal broilers inhaling ambient air or 100\% oxygen. Poultry Sci 79: 415-425. 